\title{
Qreop
}

Revista Española de Orientación y Psicopedagogía

\section{HACIA UNA ORIENTACIÓN BASADA EN EVIDENCIAS: APLICACIÓN A UN ESTUDIO SOBRE ADOLESCENTES Y REDES}

\section{TOWARDS AN EVIDENCE-BASED EDUCATIONAL GUIDANCE: APPLIED TO A STUDY ON ADOLESCENTS AND NETWORKS}

\author{
Inmaculada Asensio Muñoz ${ }^{1}$ \\ Universidad Complutense de Madrid. Facultad de Educación-Centro de Formación del \\ Profesorado. Departamento de Investigación y Psicología en Educación. Madrid, España \\ Margarita Martín Martín \\ Universidad Complutense de Madrid. Facultad de Educación-Centro de Formación del \\ Profesorado. Departamento de Investigación y Psicología en Educación. Madrid, España \\ José Antonio Bueno Álvarez \\ Universidad Complutense de Madrid. Facultad de Educación-Centro de Formación del \\ Profesorado. Departamento de Investigación y Psicología en Educación. Madrid, España
}

\section{RESUMEN}

En la actualidad, la calidad del desempeño de los orientadores depende de su competencia para obtener e interpretar información apoyada en evidencias. El desarrollo de esta competencia implica potenciar el rol del orientador como investigador e incluir en los programas de formación métodos y técnicas que le habiliten como tal. En este artículo se hacen dos propuestas concretas. Primero, se propone la revisión sistemática como método asequible y necesario de investigación secundaria para llegar a evidencias que guíen la práctica profesional y la innovación y que permitan delimitar el estado de la cuestión en cualquier tema de interés en orientación. Segundo, se plantea el uso de técnicas arborescentes en investigación primaria cuantitativa, porque se ajustan bien a la complejidad de los fenómenos que se estudian y a las características de los datos que se suelen manejar en el trabajo psicopedagógico. Dichas propuestas se ilustran con un

\footnotetext{
${ }^{1}$ Correspondencia: Inmaculada Asensio Muñoz. Correo-e: macu@ucm.es, web: http://www.ucm.es
} 
ejemplo de investigación sobre un tema de interés actual para el orientador educativo: la relación de los adolescentes con las redes sociales. Tras un acercamiento sistemático destinado a dar respuesta a tres preguntas de revisión, se realiza un estudio empírico con árboles de decisión, para identificar perfiles de control parental, con datos de 857 estudiantes de secundaria madrileños usuarios de teléfono inteligente. Los resultados indican que los padres ejercen poco control sobre el uso que sus hijos hacen de la red y aportan aspectos diferenciales con implicaciones en la acción orientadora. Se concluye acerca de la utilidad en orientación del enfoque basado en evidencias.

Palabras clave: formación del orientador, roles del orientador, adolescentes, internet, investigadores educativos.

\begin{abstract}
Currently, the quality of counselors' performance depends on their competence to obtain and interpret evidence-based information. This means enhancing the role of the counselor as a researcher including research methods and techniques in their training programs. In this article two specific proposals are made. First, systematic review is proposed as an affordable and necessary secondary research resource, to obtain evidence to guide professional practice and innovation and that allows defining the state of the art in any topic in educational guidance. Second, the use of classification techniques in quantitative primary research is proposed because it is consistent with the complexity of the phenomena being studied and with the characteristics of the data usually managed in psycho-pedagogical work. These proposals are illustrated with an example of research focused on a topic of current interest for the educational counselor: the relationship between adolescents and social networks. After a systematic approach to answer three review questions, an empirical study with decision trees is carried out to identify parental control profiles, with data from 857 Madrid high school students, smartphone users. The results indicate that parents exercise little control over the use that their children make of the networks and contribute differential aspects with implications for guidance action. In conclusion, it is clear that the evidencebased approach in guidance is useful.
\end{abstract}

Key Words: counselor roles, counselor training, adolescents, educational researchers, internet.

\title{
Cómo citar este artículo:
}

Asensio Muñoz, I., Martín Martín, M. y Bueno Álvarez, J.A. (2021). Hacia una orientación basada en evidencias: aplicación a un estudio sobre adolescentes y redes. Revista Española de Orientación y Psicopedagogía, 32(3), 23-40. https://doi.org/10.5944/reop.vol.32.num.3.2021.32554 


\section{Introducción}

En el trabajo asistencial, tanto del campo de la salud como de la educación, la relación entre la investigación científica y la práctica marca la diferencia entre las profesiones que evolucionan más o menos hacia estándares de calidad. La relación del orientador con la investigación educativa está sufriendo una evolución paralela a la definición de las funciones que este debe realizar. Aunque delimitar dichas funciones en el contexto educativo actual es complejo (Grañeras y Parras, 2009), parece claro que la acción orientadora no se limita ya a la mera tarea remedial y se acerca más a los roles de asesor (Hernández Rivero y Mederos Santana, 2018), consultor, agente del cambio (Martínez Clares, 2017), coordinador, formador de formadores, diseñador y evaluador de programas psicopedagógicos (Boza et al., 2007). A pesar de no ser la percepción más extendida, Sanz Oro (2000), al comienzo del milenio veía al orientador claramente como un investigador, como un evaluador de programas que debe incorporar la investigación en su actividad profesional para documentar y acreditar la efectividad de sus servicios. Pero, años después, en el estudio empírico realizado por Boza et al. (2007) basado en datos de encuesta, todavía se observa que los propios orientadores dan poco valor a su papel como investigadores, relegándolo a las últimas posiciones, por importancia, tiempo y preparación, en un listado de veinticinco roles. En la actualidad, esta percepción ha de replantearse, ya que la calidad en el desempeño del resto de funciones que son esenciales requiere cada vez más que el orientador desarrolle competencias de investigación relacionadas con la evaluación de necesidades, la elaboración de proyectos, la recogida y análisis de datos, la interpretación de estos y la toma de decisiones basada en evidencias o datos contrastados. Además, para estar permanentemente actualizado, el orientador tiene que ser competente como lector de publicaciones científicas, como consumidor necesario de resultados de investigación (Sanz Oro, 2000).

Entre los contenidos que suelen incluirse en los programas universitarios para formar en investigación a los futuros orientadores no es raro encontrar los procesos clásicos de los principales paradigmas. Así, por ejemplo, dentro del proceso de investigación cuantitativa es básico trabajar el concepto de significación estadística, porque se halla omnipresente en la mayoría de los trabajos científicos de los que se han de nutrir. Aunque, como indica Sanz Oro (2000), este planteamiento no es el más útil para el orientador, porque que un resultado sea estadísticamente significativo no garantiza que tenga un significado práctico. Un complemento que mejora su aplicación es el cálculo del tamaño del efecto, cuyo estudio se recomienda actualmente con carácter general. Por otra parte, dentro del proceso de investigación cualitativa el enfoque de la investigación-acción es claramente idóneo en la formación de orientadores como investigadores, aunque una opción que está ganando cada vez más partidarios, por razones de pragmatismo, son los métodos mixtos (Johnson y Onwuegbuzie, 2004).

En este artículo se plantean e ilustran dos herramientas metodológicas escasamente presentes en los programas de formación de orientadores que, sin embargo, pueden ser especialmente adecuadas para atender a alguno de los principales desafíos de la investigación y de la intervención que se realiza actualmente en orientación y de la retroalimentación necesaria entre ambas. Situándose en cualquiera de los paradigmas, un reto al que se enfrenta el orientador en nuestros días es disponer de información clara, fiable y relevante acerca del estado de la cuestión en el que se encuentra el tema sobre el que tiene que intervenir o seguir investigando. Otro desafío, presente cuando se trabaja con metodología cuantitativa o mixta, es contar con técnicas estadísticas multivariadas de análisis de datos que permitan una fácil y rápida interpretación, con significación práctica además de estadística, que cumplan los requisitos de ser adecuadas al tipo de problema planteado y ajustadas a la complejidad de la realidad que se estudia y a las características de los datos que se analizan. 
En cuanto al primer reto, hay que señalar que la productividad científica en educación es profusa, como en cualquier campo, por el desarrollo incremental que han experimentado los medios de comunicación. Pero, la revisión del estado de la cuestión, que es una fase esencial en el proceso de investigación científica, se sigue afrontando con frecuencia sin un esquema metodológico que permita garantizar que la investigación anterior se sintetiza adecuadamente. Incorporar la revisión sistemática a los modos habituales de proceder en investigación educativa es tan importante que puede suponer el que por fin se llegue a caminar en educación como se camina en otras disciplinas científicamente más avanzadas, sobre hombros de gigantes. La evidencia que se genera a partir de la acumulación y síntesis de resultados de investigaciones primarias es necesaria para que los profesionales tengan datos contrastados en los que basar sus prácticas y para que los investigadores dispongan de un estado de la cuestión fiable que permita discernir lo que ya se sabe sobre el tema y lo que la investigación que se realiza a partir de ese momento puede aportar como original. En el ámbito sanitario, este tipo de postulados están asentados y forman ya parte de una cultura, compartida por toda la comunidad científica, en la que la publicación de síntesis de investigación se ajusta a protocolos tan elaborados como PRISMA (Shamseer et al., 2015). El auge de revisiones sistemáticas y metaanálisis en ciencias de la salud está muy ligado al impulso de redes internacionales de investigación como la Colaboración Cochrane (Camilli, 2015) y la medicina basada en la evidencia cuenta con una vasta tradición. En nuestro ámbito, cabe destacar la labor que realiza The Evidence for Policy and Practice Information and Coordinating Centre (EPPI-Centre), que está haciendo contribuciones metodológicas importantes y realizando revisiones sistemáticas en una gran cantidad de temas relacionados con la educación (Blanco, 2012). Sin embargo, a pesar de que cada vez se va imponiendo más, la revisión sistemática todavía no es de uso frecuente en nuestro campo y no se tiene apenas en cuenta en los programas de formación de orientadores. Entendemos que responder a este desafío es necesario al tiempo que viable, si, reservando la metodología más compleja de metaanálisis para el nivel de doctorado, en los programas universitarios orientados a la formación de profesionales se enseñan metodologías como la propuesta por Andrews y Harlen (2006), que sirve de marco para el ejemplo que presentaremos aquí.

Con referencia al segundo reto, hay que advertir que los fenómenos educativos que son objeto de interés en orientación tienen como principal característica su complejidad, en el sentido de que en ellos intervienen simultáneamente múltiples variables de diferente naturaleza que interaccionan entre sí. Sin embargo, en los cursos universitarios se aprenden normalmente las técnicas de análisis de datos más simples o algunas técnicas avanzadas, muy exigentes y poco intuitivas, que precisamente por eso no resultan demasiado prácticas. El orientador, como investigador en la acción o como profesional que requiere de información contrastada, para la evaluación de necesidades en su centro o para la evaluación de los programas que implementa, debe disponer de herramientas más adecuadas. La alternativa que aquí presentamos, como asequible para un orientador que dispone de bases de datos amplias, de un centro o de varios, son los árboles de decisión, que son unas técnicas de segmentación cuyo propósito es "dividir las poblaciones o muestras objeto de estudio, siguiendo un proceso secuencial descendente, para la identificación de subgrupos homogéneos en una variable de interés para el investigador" (Blanco Blanco et al., 2017, p. 237).

Tras la argumentación expuesta, el objetivo general de este trabajo es contribuir a mejorar el utillaje de trabajo del orientador, proponiendo y ejemplificando dos metodologías actuales, viables y útiles, que le ayudarán a integrar la investigación en su quehacer profesional como medio de informar la práctica y de avanzar en la resolución de problemas que afectan a su saber y su saber hacer. Específicamente se pretende ilustrar: 1) un procedimiento sencillo para llegar a una síntesis de investigación mediante un proceso de revisión sistemática y 2) la facilidad de uso e interpretación de los árboles de decisión, como técnicas multivariadas de obtención de perfiles basadas en análisis de segmentación que incorporan la detección de la interacción.

La metodología de revisión sistemática y los árboles de decisión son aplicables a prácticamente todos los temas propios de la orientación. El tópico seleccionado para el ejemplo que se desarrolla aquí ha sido "el estudio de la relación de los adolescentes con las redes sociales 
en España", por ser un contenido de interés para el orientador, que muchas veces tiene que prevenir o afrontar problemas de convivencia o de rendimiento relacionados con el mal uso o abuso que los estudiantes de secundaria puedan llegar a hacer de las redes sociales (Martín Martín et al., 2021). La elección del tema además se basa en la disponibilidad de una base de datos actualizados para aplicar una herramienta de clasificación como la que se pretende ilustrar (Martín Martín, 2015). Por otra parte, aunque se han hecho revisiones sistemáticas relacionadas con este tema, son por lo general de carácter clínico (Parada Rico y García Suárez, 2017; Rojas Jara et al., 2018; Rubiales et al., 2018; Vega Almeida y Arencibia Jorge, 2019, entre otras), por lo que resulta pertinente una revisión desde una perspectiva psicopedagógica. La revisión que se presenta tiene como objetivo dar respuesta a tres preguntas: a) ¿en qué contextos se realiza la investigación sobre la relación de los adolescentes con las redes sociales en España?, b) ¿cuáles son los métodos de investigación empleados? y c) ¿qué temas de interés emergen para la acción orientadora?

Por otra parte, centrándonos en estudios cuantitativos o mixtos y dejando, por tanto, a un lado los trabajos que se hacen con metodología cualitativa (Calvo y San Fabián, 2018; Pérez Escoda y Contreras, 2018; Ruido et al., 2017), la investigación realizada en el ámbito de la relación de los adolescentes con las redes sociales en España no suele hacerse desde una perspectiva multivariada. Siendo muy interesantes, la mayoría de estos estudios se quedan en una descripción de cada variable por separado o en el estudio de la relación entre una variable independiente y otra dependiente, mediante el uso de los coeficientes de correlación y de las pruebas de contraste más habituales (Alonso Ruido et al., 2015; Cabero et al., 2016; Colás Bravo et al., 2017; Giménez et al., 2017...). Dado que la revisión pone de manifiesto el uso mayoritario de enfoques univariados o bivariados y, siendo más apropiada para el estudio de fenómenos educativos una aproximación que permita captar e interpretar las interacciones, se proponen los árboles de decisión como herramienta para dar respuesta al problema de investigación que hemos elegido de ejemplo: ¿qué perfiles de control parental emergen en el estudio de la relación de los adolescentes con las redes sociales? La elección específica del tópico "control parental" se justifica por la importancia que este tema tiene desde el punto de vista educativo, primero, por el bajo nivel de supervisión parental que resulta de los estudios, y, segundo, por la relación que ello tiene con algunos peligros que desde los programas de orientación tendría interés considerar. La propuesta del enfoque se basa en que los árboles de decisión se ajustan a la naturaleza diversa de los datos con los que se suele trabajar en educación, muchas veces procedentes de cuestionarios elaborados ad hoc. Además, los árboles son más adecuados, por su versatilidad, que otras aproximaciones multivariadas más frecuentes, como la regresión logística (Malo et al., 2018) o los modelos de ecuaciones estructurales (González et al., 2017). A pesar de ello, estas técnicas de clasificación, que se emplean habitualmente en investigación de mercado (Blanco Blanco et al., 2017), apenas están empezando a imponerse en investigación educativa (Arroyo Resino et al., 2019; Asensio Muñoz et al., 2018; Lizasoain y Joaristi, 2000; López Martín et al., 2018).

\section{Método}

Para el logro del primer objetivo y dar respuesta a las tres preguntas que guían la revisión, se realiza una revisión sistemática elemental, en la que se parte del esquema metodológico que proponen Andrews y Harlen (2006), poniendo énfasis en las fases de justificación de la necesidad de la revisión, planteamiento de las preguntas de revisión ("review questions"), identificación de los criterios de inclusión/exclusión, búsqueda de documentos en bases de datos científicas electrónicas y selección y exploración de los estudios con el fin de dar respuesta a las preguntas 
planteadas. La revisión se justifica por la gran cantidad de documentos publicados sobre el tema, que hace que definir de manera fiable el estado de la cuestión se plantee como una necesidad. Las preguntas de revisión a las que se pretende dar respuesta no se refieren tanto a resultados, como a delimitación del contexto y de los métodos y temas que se suelen investigar en este campo. Por razones de espacio, el interés de la búsqueda se limita al estudio de las relaciones que tienen los adolescentes con las redes sociales digitales en nuestro entorno geográfico más cercano. En consecuencia, se han elegido las bases de datos del Consejo Superior de Investigaciones Científicas (CSIC), de acceso libre, que contienen la producción científica publicada en España desde la década de los 70. ÍnDICEs-CSIC es un recurso bibliográfico multidisciplinar que recopila y difunde fundamentalmente artículos de investigación publicados en revistas científicas españolas (https://indices.csic.es/).

Para conseguir el segundo objetivo y responder a la pregunta de investigación, se realiza un estudio cuantitativo con finalidad exploratoria, que se basa en los resultados de un cuestionario elaborado "ad hoc" (Martín Martín, 2015), de 14 ítems que se refieren a diferentes dimensiones de las relaciones de los adolescentes con las redes ( $\alpha=0,716$, en un intervalo confidencial al $95 \%$ entre 0,685 y 0,745 ) y 6 preguntas criterio (tablas 1 y 2), que se aplica a 857 adolescentes con edades comprendidas entre los 14 y 16 años, matriculados en centros educativos de Madrid que aceptaron participar en la investigación. Mediante IBM SPSS Statistics 25, se obtuvieron estadísticos descriptivos y el árbol de decisión, introduciendo en el modelo de clasificación las preguntas que se incluyen en las tablas 1 y 2 , junto con otras variables como: edad, edad de apertura de la primera red social, tipo de centro educativo, tener o no ordenador en casa y en clase, preferir el móvil o el ordenador, género, curso, origen español/inmigrante, país de origen, importancia que dan a las redes sociales, qué redes sociales tienen y cuál usan más). El algoritmo utilizado fue CHAID (Chi Automatic Interaction Detection) (Kass, 1980).

\section{Tabla 1}

Preguntas, escala de respuesta de cada ítem y correlación del ítem con el total corregido

\begin{tabular}{|c|c|c|c|}
\hline CUESTIONARIO & Mínimo & Máximo & $\mathrm{IH}$ \\
\hline $\begin{array}{l}\text { 1.- Agrego a mis contactos a todos los que quieran ser mis amigos, } \\
\text { aunque no los conozca }\end{array}$ & 1 (nunca) & 5(siempre) & 296 \\
\hline 2.- Converso con todos los contactos que tengo en mi lista & 1 (nunca) & 5(siempre) & criterio \\
\hline $\begin{array}{l}\text { 3.- En mi perfil hablo de mis estados de ánimo, sentimientos, gustos, } \\
\text { garejas, etc. }\end{array}$ & 1 (nunca) & 5 (siempre) & ,401 \\
\hline $\begin{array}{l}\text { 4.- Me he sentido mal por comentarios o fotos colgadas en la red en las } \\
\text { que salgo poco favorecido }\end{array}$ & 1 (nunca) & 5 (siempre) & 187 \\
\hline $\begin{array}{l}\text { 5.- Soy igual de directo diciendo las cosas a través de la red que en } \\
\text { persona }\end{array}$ & 1 (nunca) & 5(siempre) & criterio \\
\hline $\begin{array}{l}\text { 6.- Lo que pasa en la red social con mis amigos me afecta en la forma de } \\
\text { tratarlos después en persona }\end{array}$ & 1 (nunca) & 5 (siempre) & ,239 \\
\hline $\begin{array}{l}\text { 7.- Elijo Internet cuando tengo algo comprometido que decirle a alguien } \\
\text { (sentimientos, un problema...) }\end{array}$ & 1 (nunca) & 5 (siempre) & 352 \\
\hline $\begin{array}{l}\text { 8.- Soy capaz de saber cómo se está sintiendo la persona con la que } \\
\text { estoy chateando }\end{array}$ & 1 (nunca) & 5 (siempre) & ,342 \\
\hline $\begin{array}{l}\text { 9.- Prefiero chatear con mis amigos habituales que hacerlo con gente a la } \\
\text { que no suelo ver con mucha frecuencia }\end{array}$ & 1 (nunca) & 5 (siempre) & criterio \\
\hline .- He ten & ca) & $5(\mathrm{sic}$ & 256 \\
\hline $\begin{array}{l}\text { 11.- Mientras estoy conectado a la red social realizo otras actividades, } \\
\text { como estudiar o ver la televisión }\end{array}$ & 1 (nunca) & 5(siempre) & ,316 \\
\hline 12.- Mis amigos de la red, a los que no suelo ver en persona, confían en & 1 (nunca) & 5 (siempre) & ,331 \\
\hline 13.- La red me ayuda a expresa & 1 (nunca) & 5 (siempre) & 486 \\
\hline 14.- Mi vida sentimental y social ha mejorado con las redes sociales & 1 (nada) & 4(mucho) & ,421 \\
\hline
\end{tabular}




\section{Tabla 2}

Preguntas, escala de respuesta de cada ítem y correlación del ítem con el total corregido (Continuación)

\begin{tabular}{|c|c|c|c|}
\hline CUESTIONARIO & Mínimo & Máximo & $\mathrm{IH}$ \\
\hline 15.- Mis padres controlan mi uso de las redes sociales & 1(nada) & \multicolumn{2}{|c|}{ 4(mucho) criterio } \\
\hline $\begin{array}{l}\text { 16.- Tengo restringido el acceso a mi perfil (PRIVADO) para que solo lo } \\
\text { vean mis amigos }\end{array}$ & $0($ no) & 1 (sí) & criterio \\
\hline 17.- Conozco mejor a la gente que me rodea gracias a Internet & $0(\mathrm{no})$ & 1 (sí) & ,346 \\
\hline 18.- La frecuencia con la que me conecto a las redes sociales es de ... & $\begin{array}{l}1 \text { (menos de } 1 \\
\text { vez semana) }\end{array}$ & 4 (a diario) & 361 \\
\hline $\begin{array}{l}\text { 19.- El tiempo que dedico a las redes sociales cada vez que me conecto } \\
\text { es de ... }\end{array}$ & 1 (media hora) & $\begin{array}{c}5 \text { (más de } \\
3 \text { horas) }\end{array}$ & ,388 \\
\hline 20.- El tiempo que dedico a estudiar cada día es de ... & 1 (media hora) & $\begin{array}{c}5 \text { (más de } \\
3 \text { horas) }\end{array}$ & criterio \\
\hline
\end{tabular}

\section{Resultados}

\section{Estado de la cuestión resultante del proceso de revisión sistemática}

Se inicia la investigación secundaria con una búsqueda simple usando los términos "adolescentes y redes sociales". De esta búsqueda salen 197 documentos, de los que, filtrando los más actuales (2016-2019) y utilizando los criterios de inclusión/exclusión que se resumen en la figura 1, terminan seleccionándose 14 (tabla 3), con una coincidencia Inter jueces del 82,15\%.

Respondiendo a la primera pregunta de revisión, los artículos analizados representan una amplia parte del territorio nacional, en el que Cataluña y Andalucía son las Comunidades mejor representadas (28,58\%), seguidas de Galicia (21,43\%) y de Madrid, Aragón y Castilla León $(14,3 \%$ cada una). Solo hay un estudio con muestra de Baleares, Canarias, País Vasco, de Valencia y de La Rioja $(0,07 \%$ cada una). Por otra parte, hay 2 estudios que abarcan más de una Comunidad Autónoma (14,3\%).

Con relación a la segunda pregunta de revisión, centrada en la metodología, se observa que el $57,14 \%$ son estudios cuantitativos, el $21,43 \%$ son cualitativos y el otro $21,43 \%$ son mixtos. Esto significa que, según la revisión realizada, el enfoque cuantitativo sigue siendo el más frecuente en la investigación que se publica en revistas científicas en España sobre el tema estudiado. El instrumento de recogida de datos más usado es el cuestionario, que, en sus diferentes modalidades, estandarizado o ad hoc, se emplea en toda la investigación cuantitativa y mixta que ha surgido de la selección (78.57\%). En cuanto a las técnicas estadísticas de análisis de datos destacan como las más complejas el análisis de ecuaciones estructurales, análisis multivariado de la varianza (MANOVA) y el análisis factorial exploratorio y confirmatorio (AFE y AFC) (González Sanmamed et al., 2017; Oberst et al., 2016; Romera et al., 2016).

Por último, atendiendo a la tercera pregunta de revisión, los temas se centran en: factores que motivan el uso de las redes sociales por los estudiantes, seguridad y riesgos (42,86\%), el género 
en la red $(21,43 \%)$, la participación social y ocio en entornos digitales $(21,43 \%)$ y el papel de padres, profesores y tutores en la relación de los adolescentes con las redes (14,28\%) (López De Ayala et al., 2019; Prats et al., 2018). Este último es el tópico más vinculado al campo del trabajo del orientador y el que se trata específicamente en el estudio cuantitativo, cuyos resultados se presentan a continuación.

\section{Figura 1}

Diagrama de flujo del proceso de selección de artículos

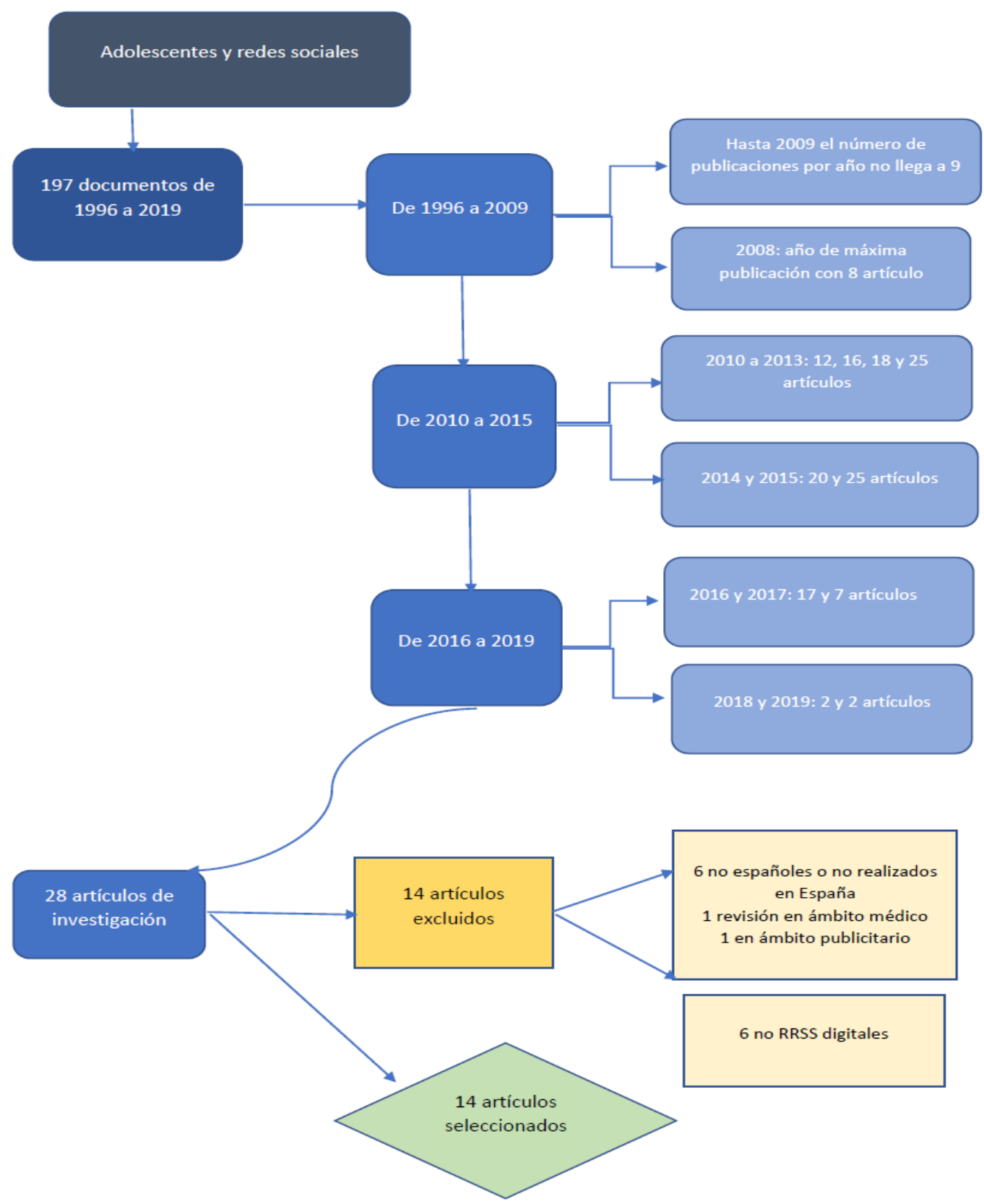

Fuente: Elaboración propia 


\section{Tabla 3}

Artículos que cumplen los criterios de inclusión en el proceso de revisión sistemática

\begin{tabular}{|c|c|c|c|}
\hline Referencia (*) & Contexto & Método & Tema \\
\hline $\begin{array}{l}\text { Martín Montilla et } \\
\text { al. (2016) }\end{array}$ & $\begin{array}{l}511 \text { sujetos entre } 15 \\
\text { y } 19 \text { años, de } 6 \text { IES } \\
\text { Huelva y Granada }\end{array}$ & $\begin{array}{c}\text { MIXTO } \\
\text { Cuestionarios, grupo de } \\
\text { discusión y análisis de } \\
\text { producciones escritas. }\end{array}$ & $\begin{array}{l}\text { Violencia de género y } \\
\text { redes }\end{array}$ \\
\hline Oberst et al. (2016) & $\begin{array}{l}623 \text { sujetos entre } 12 \\
\text { y } 16 \text { años de } \\
\text { Cataluña }\end{array}$ & $\begin{array}{l}\text { CUANTITATIVO } \\
\text { Cuestionarios. t, MANOVA, } \\
\text { eta }^{2}, \text { Correlaciones }\end{array}$ & $\begin{array}{l}\text { Estereotipos de } \\
\text { género en la red }\end{array}$ \\
\hline Pavón et al. (2016) & $\begin{array}{c}852 \text { sujetos entre } 12 \\
\text { y } 16 \text { años, de } \\
\text { Gipuzkoa }\end{array}$ & $\begin{array}{l}\text { MIXTO. } \\
\text { Cuestionarios, entrevistas en } \\
\text { profundidad y análisis de } 16 \\
\text { perfiles en Tuenti. }\end{array}$ & $\begin{array}{l}\text { Consumo audiovisual, } \\
\text { contenidos de ficción } \\
\text { televisiva. }\end{array}$ \\
\hline Plaza (2016) & $\begin{array}{c}1032 \text { sujetos entre } 12 \\
\text { y } 17 \text { años de } \\
\text { Andalucía, Aragón, } \\
\text { Cataluña, Castilla- } \\
\text { León y Madrid }\end{array}$ & $\begin{array}{c}\text { CUANTITATIVO } \\
\text { Cuestionarios } \\
\%\end{array}$ & $\begin{array}{c}\text { Uso de la red } \\
\text { (motivación, papel en } \\
\text { el aprendizaje, } \\
\text { peligros...) }\end{array}$ \\
\hline $\begin{array}{l}\text { Ricoy y Fernández } \\
\text { Rodríguez (2016) }\end{array}$ & $\begin{array}{c}95 \text { sujetos entre } 13 \text { y } \\
17 \text { años, de zona } \\
\text { urbana de Galicia }\end{array}$ & $\begin{array}{c}\text { CUALITATIVO } \\
\text { Técnica de relato (AQUAD) }\end{array}$ & $\begin{array}{l}\text { Prácticas de ocio en } \\
\text { juventud }\end{array}$ \\
\hline $\begin{array}{c}\text { Rodríguez García y } \\
\text { Magdalena } \\
\text { Benedito (2016) }\end{array}$ & $\begin{array}{l}170 \text { sujetos entre } 12 \\
\text { y } 16 \text { años de } 3 \\
\text { municipios de } \\
\text { Valencia }\end{array}$ & $\begin{array}{c}\text { CUANTITATIVO } \\
\text { Cuestionarios. \%, Gráficas de } \\
\text { barras y sectores }\end{array}$ & $\begin{array}{c}\text { Seguridad y } \\
\text { privacidad en la red }\end{array}$ \\
\hline Romera et al. (2016) & $\begin{array}{l}505 \text { sujetos entre } 12 \\
\text { y } 16 \text { años de } 2 \\
\text { centros de Andalucía }\end{array}$ & $\begin{array}{c}\text { CUANTITATIVO } \\
\text { Cuestionarios } \\
\text { AFE, AFC, pruebas H y U }\end{array}$ & Ciberbullying \\
\hline $\begin{array}{l}\text { Sabater Fernández } \\
(2016)\end{array}$ & $\begin{array}{c}400 \text { sujetos entre } 14 \\
\text { y } 20 \text { años, de La } \\
\text { Rioja }\end{array}$ & $\begin{array}{c}\text { MIXTO } \\
\text { Entrevistas semiestructuras y } \\
\text { cuestionarios (Chi², ANOVA, Phi } \\
\text { de Kramer...) }\end{array}$ & $\begin{array}{c}\text { Configuración de la } \\
\text { intimidad tradicional y } \\
\text { en la red }\end{array}$ \\
\hline $\begin{array}{l}\text { De Frutos Torres y } \\
\text { Marcos Santos } \\
\text { (2017) }\end{array}$ & $\begin{array}{c}370 \text { sujetos entre } 14 \\
\text { y } 18 \text { años, de } 4 \\
\text { centros de la ciudad } \\
\text { de Segovia }\end{array}$ & CUANTITATIV́O & $\begin{array}{l}\text { Percepción de riesgos } \\
\text { en la red (uso, } \\
\text { incidentes, ...) }\end{array}$ \\
\hline $\begin{array}{c}\text { González } \\
\text { Sanmamed et al. } \\
(2017)\end{array}$ & $\begin{array}{l}1144 \text { sujetos entre } 15 \\
\text { y } 18 \text { años, de } 29 \\
\text { centros de A Coruña }\end{array}$ & $\begin{array}{l}\text { CUANTITATIVO } \\
\text { Cuestionarios } \\
\text { Ecuaciones estructurales con } \\
\text { mínimos cuadrados parciales }\end{array}$ & $\begin{array}{l}\text { Uso que hacen los } \\
\text { adolescentes de la red }\end{array}$ \\
\hline $\begin{array}{l}\text { Villanueva Baselga } \\
\text { et al. (2017) }\end{array}$ & $\begin{array}{l}1709 \text { sujetos de } \\
\text { cuarto de ESO de } \\
\text { Cataluña }\end{array}$ & $\begin{array}{c}\text { CUANTITATIVO } \\
\text { Cuestionarios. \%, medias, } \\
\text { desviaciones, correlaciones }\end{array}$ & $\begin{array}{l}\text { Vinculación entre uso } \\
\text { de medios y redes e } \\
\text { interés político }\end{array}$ \\
\hline $\begin{array}{l}\text { Donoso Vázquez et } \\
\text { al. (2018) }\end{array}$ & $\begin{array}{c}3043 \text { sujetos de } 3^{\circ} \text { y } \\
\text { 4ํo de ESO, de } \\
\text { Andalucía, Aragón, } \\
\text { Baleares, Canarias, } \\
\text { Cataluña, Galicia }\end{array}$ & $\begin{array}{c}\text { CUANTITATIVO } \\
\text { Cuestionarios }\end{array}$ & $\begin{array}{c}\text { Violencia de género } \\
2.0\end{array}$ \\
\hline Prats et al. (2018) & $\begin{array}{l}1200 \text { sujetos de } 8 \\
\text { centros de ESO y } 1^{\circ} \\
\text { de Bachiller, de } \\
\text { Cataluña }\end{array}$ & $\begin{array}{c}\text { INNOVACIÓN/CUALITATIVO } \\
\text { Aplicación piloto y observación } \\
\text { asistemática }\end{array}$ & $\begin{array}{l}\text { Taller sobre uso } \\
\text { adecuado de la red }\end{array}$ \\
\hline $\begin{array}{c}\text { López De Ayala et } \\
\text { al. (2019) }\end{array}$ & $\begin{array}{c}26 \text { padres de } \\
\text { adolescentes de } 9 \text { a } \\
17 \text { de la Comunidad } \\
\text { de Madrid }\end{array}$ & $\begin{array}{c}\text { CUALITATIVO } \\
\text { Entrevistas en profundidad }\end{array}$ & $\begin{array}{l}\text { Uso que hacen los } \\
\text { hijos de la red }\end{array}$ \\
\hline
\end{tabular}




\section{Obtención de perfiles de control parental mediante árboles de decisión}

Las respuestas al cuestionario de los 857 adolescentes indican que son usuarios de teléfono inteligente, con una edad media de 15,02 ( $D M=0,782)$, escolarizados en su mayoría en tercero y cuarto de Educación Secundaria Obligatoria (ESO) (51,9\% y 38,3\% respectivamente) y en Programas de Cualificación Profesional Inicial (PCPI) (9,7\%). Del total, un 62,3\% estudian en centros públicos, un $28,9 \%$ en concertados y el resto en colegios privados $(8,8 \%)$. En cuanto a género, la distribución es equilibrada, aunque hay un porcentaje algo inferior de varones (47\%). Aproximadamente el $76,7 \%$ de los participantes son españoles y hay un $23,3 \%$ de origen inmigrante de 26 nacionalidades diferentes, siendo el segundo país de origen más numeroso Ecuador, el tercero Colombia y el cuarto República Dominicana. El 63,7\% de la muestra prefiere el móvil para conectarse, mientras que el $24,7 \%$ prefiere hacerlo con el ordenador y el resto no manifiesta una preferencia clara (11,6\%). Más de la mitad de los encuestados dan importancia al hecho de pertenecer a una red social $(54,1 \%)$ y la mayoría pertenecen a más de una $(89,4 \%)$, siendo el valor más frecuente pertenecer a tres (36,3\%). Sólo un $0,2 \%$ de participantes manifiestan no pertenecer a ninguna red social. Las percepciones que estos adolescentes tienen del control parental se ofrecen en la figura 2. El $37 \%$ de ellos piensa que sus padres no les controlan en absoluto, y cerca de un $40 \%$ responden que les controlan poco. Los que perciben que sus padres les controlan bastante son 148 sujetos, que representan un $17,3 \%$ de los consultados. Por último, un 5,7\% contestan que sus padres les controlan mucho.

\section{Figura 2}

Porcentajes de respuesta en el ítem de control parental

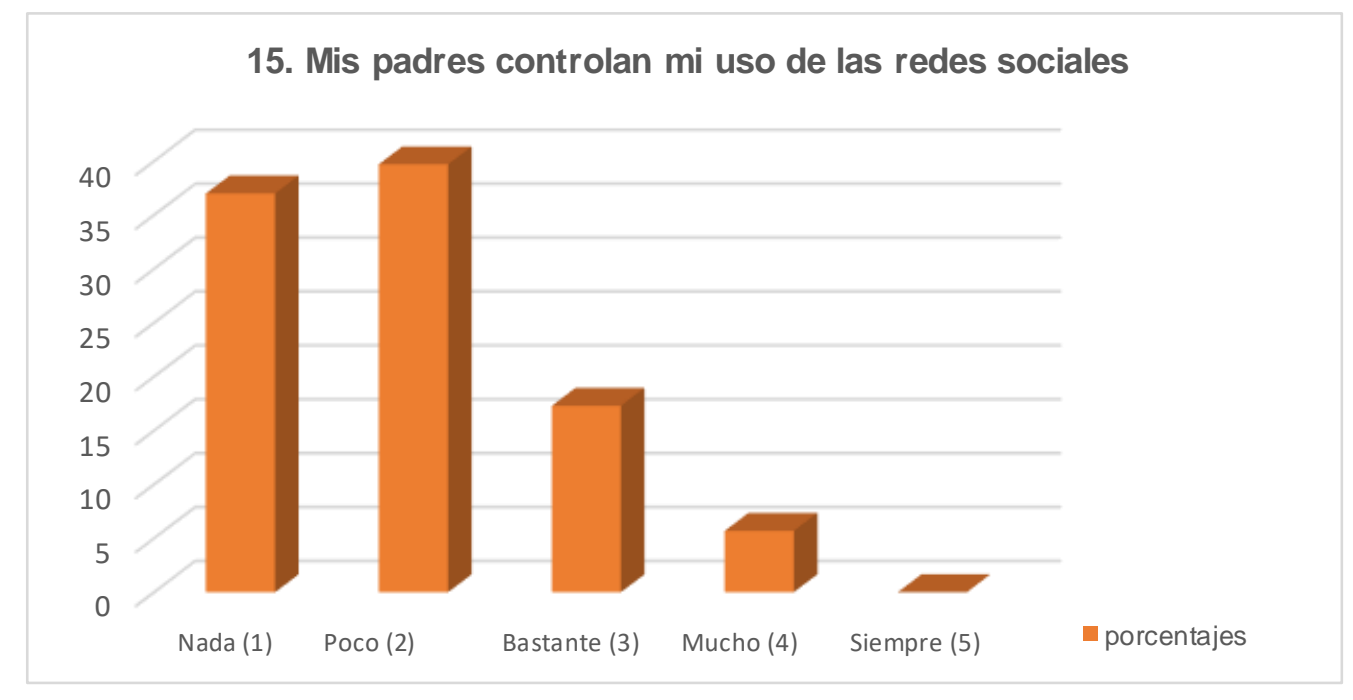

Fuente: Elaboración propia

Con CHAID se obtiene el diagrama de árbol (figura 3) con los perfiles de grado de control percibido (ítem 15) que corresponde al criterio seleccionado para este análisis como variable de segmentación. Se parte de un nodo 0 en el que la media en control parental es 1,917, esto es, no llega a 2 en una escala de 1 a 5 , y la desviación típica es 0,876 , con 3 valores perdidos $(\mathrm{N}=854)$. La variable más discriminativa, que subdivide al grupo total en tres grupos o ramas del árbol, es la 20 (tiempo dedicado al estudio), que interactúa con la 11 (actividad multitarea en la que el punto de corte se sitúa en 4), la edad (estando el punto de corte en los 15 años) y la edad de apertura de la primera red social (con puntos de corte en los 10 y en los 12 años). Los nodos más extremos son el 9 y el 5 . En el 9 se agrupan 443 adolescentes con padres percibidos como más 
controladores (Media=2,138), que se caracterizan por ser los más estudiosos y centrados en la tarea. El nodo 5 reúne a los mayores de 15 años y a su vez menos estudiosos, que es el grupo que se asocia con la media menor en nivel de control percibido $(1,32)$. El uso que hacen de las redes (variable 11) solo discrimina entre los sujetos que más estudian (nodo 3 ). Entre los sujetos del nodo 2, la variable que discrimina es la edad de apertura de la primera red, de modo que los que tienen padres que ejercen un mayor control son también los más tardíos: abren su primera red con más de 12 años. Entre los que menos estudian (nodo 1) la única variable que produce diferencias significativas $(p=0,017)$ es la edad, que da lugar a los nodos 4 y 5 . En suma, se observa que la edad, edad de apertura de la primera red y la actividad multitarea emergen como variables que interactúan en la relación tiempo de estudio y control parental de diferente manera para quienes estudian media hora al día (nodo 1), los que estudian entre una hora y dos (nodo 2) y los que estudian más de 2 horas (nodo 3). La correlación de Spearman entre las variables 15 y 20 es de $0,259(p=0,000)$.

\section{Figura 3}

Perfiles de grado de control percibido en la muestra de adolescentes con Smartphone

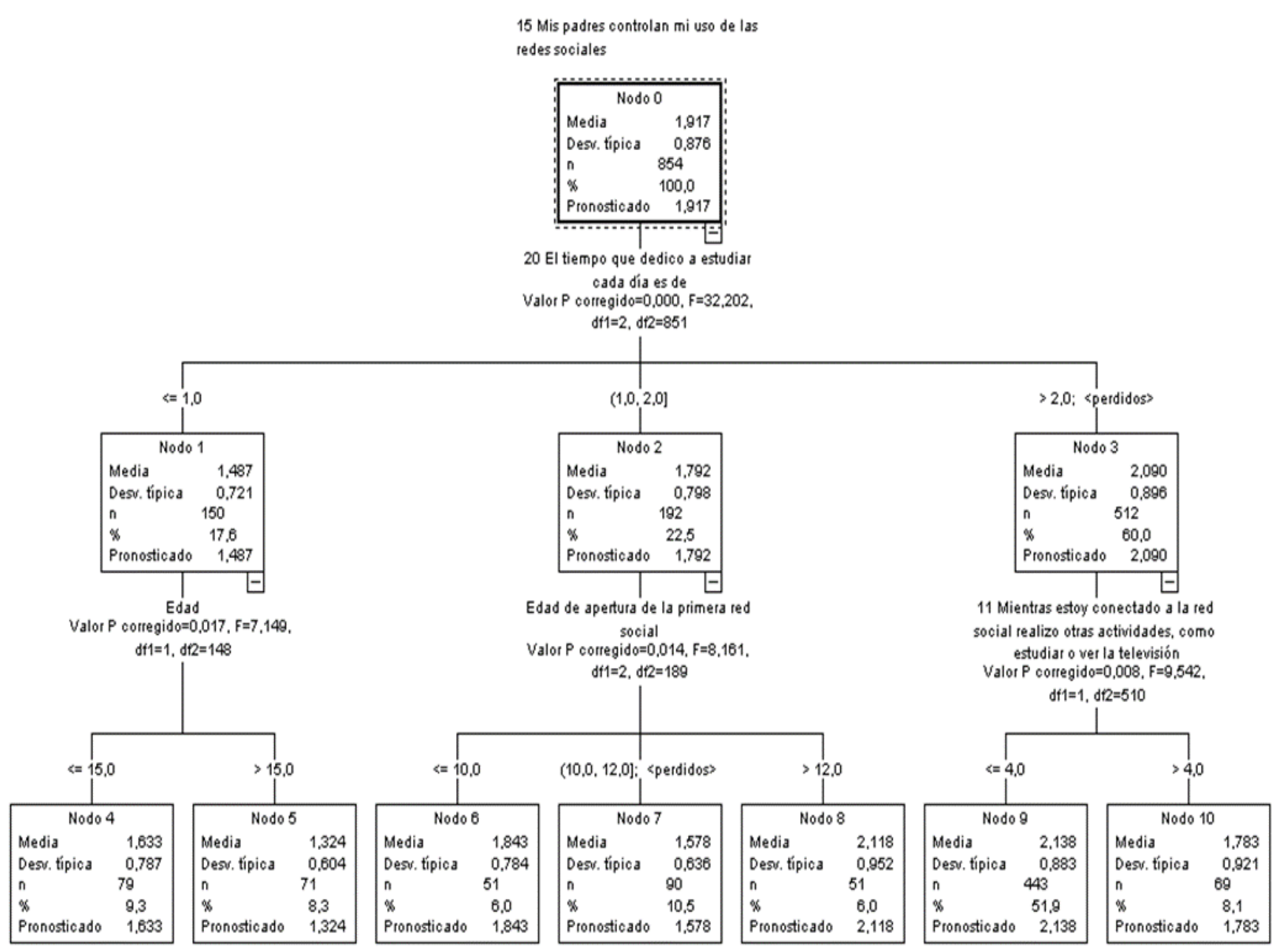

Fuente: Elaboración propia 


\section{Conclusiones y Discusión}

Atendiendo a los objetivos planteados, se ha ilustrado la adecuación de dos estrategias, la revisión sistemática y los árboles de decisión, que se presentan como recursos novedosos que el orientador debe ir incorporando a su bagaje profesional, para apoyarse en evidencias. Junto con otros, que ya forman parte de su caja de herramientas profesional, estos instrumentos de trabajo, convenientemente usados, le permitirán un contacto más estrecho 1) con la comunidad científica de orientadores y 2) con la realidad educativa, como fuentes principales de obtención de datos fiables e información contrastada.

Se trata de un artículo que pretende poner en valor el rol del orientador como investigador, por lo que tiene un carácter procedimental y sería aplicable a diferentes contenidos relacionados con la orientación. El tema del ejemplo se ha seleccionado por su pertinencia y actualidad, dado que es un hecho que internet ha entrado de lleno en la vida de los adolescentes (Álvarez García et al., 2019) y los programas de orientación en ESO y PCPI deben estar atentos al estudio de sus efectos, para la prevención y la formación de los jóvenes y sus familias en un uso responsable y eficiente de las redes sociales (Sobrado et al., 2013). La orientación, que ha de estar atenta a las necesidades educativas emergentes, tiene claramente, en la proliferación del uso de los dispositivos inteligentes en la sociedad líquida en la que vivimos, un nuevo campo de desarrollo (Ramírez García et al., 2020).

Los resultados de la revisión sistemática, a pesar de haberse limitado a una única base bibliográfica, responden a las preguntas planteadas y demarcan el interés del tema elegido, que, en el periodo estudiado, se realiza con muestras que abarcan prácticamente todo el territorio nacional. De la síntesis se obtiene además que los métodos que se emplean en la investigación sobre el tema son diversos, predominando los estudios descriptivos univariados y bivariados basados en datos de cuestionarios. Emergen también diferentes centros de interés entre los cuales se encuentra el control parental. Los resultados del estudio empírico con árboles de decisión, que profundiza en este tópico, indican que los padres ejercen por lo general poco o ningún control sobre el uso que sus hijos hacen de la red y van en la línea de los obtenidos en los estudios seleccionados en el proceso de revisión sistemática relacionados con la temática, hechos desde la perspectiva de los padres. Otros estudios que ponen de manifiesto la escasa supervisión que tanto chicos como chicas tienen de sus progenitores cuando acceden y hacen uso de las redes sociales son los de Alonso-Ruido et al. (2015) o Fernández et al. (2015). Gomes y Sendín, (2014) Ilegan a resultados similares e informan de que los padres establecen límites al consumo de contenidos televisivos inapropiados, pero, que, cuando sus hijos están conectados a internet a través del ordenador, este control disminuye. Aunque Giménez et al. (2017) y Villanueva Blasco y Serrano Bernal (2019) obtienen que las hijas están algo más controladas que los hijos. Estos hallazgos son relevantes desde el punto de vista psicopedagógico, ya que existe relación significativa entre la escasez de supervisión familiar y la implicación en dinámicas de ciberacoso y de posible ciberadicción. Además, en el caso de las chicas, la restricción de horarios nocturnos de conexión a internet aplicada a través del control parental es una medida eficaz para reducir la probabilidad de exposición a solicitudes de sexting y, en consecuencia, de poder ser víctima de ciberbullying o sextorsión. Por otro lado, el árbol de decisión aporta información relevante para padres, tutores y orientadores acerca de la interacción que existe entre el control parental del uso de redes y el número de horas de estudio. Según el tipo de estudiante, intervienen otras variables en el perfil, como la edad, la edad de apertura de la primera red y la realización de otras tareas mientras se está conectado a la red. Los nodos que surgen del análisis de clasificación permiten específicamente al orientador identificar los grupos más necesitados de prevención y formación. Los resultados de la investigación empírica avalan la necesidad de insistir en el control parental 
tanto en los procesos de orientación familiar como en las escuelas de padres, en las que se pueden atender sus demandas de información acerca de cómo aplicar límites y horarios de uso de la tecnología e internet (Prats et al., 2018).

Aunque estos resultados aportan información de interés para la toma de decisiones en la acción orientadora, más allá de la respuesta a la cuarta pregunta o problema de investigación, en este trabajo se presenta una propuesta innovadora que consiste en incorporar en el "utillaje" profesional del orientador dos herramientas concretas, la revisión sistemática y los árboles de decisión. Se aboga, en consecuencia, por la necesidad de actualización en la formación de los orientadores en su faceta de investigadores educativos, que, cada vez con más claridad, entra a formar parte de su perfil profesional en el siglo XXI (Martínez Clares, 2017), en el convencimiento de que la formación universitaria de los orientadores, para ser de calidad, debe ser, además de eficaz y eficiente, funcional (Orden et al., 2002), lo que supone estar atentos a las nuevas necesidades e incluir contenidos y desarrollar competencias que les permitan satisfacerlas adecuadamente.

Centrar el foco de atención en estos recursos metodológicos se argumenta en torno a la infrecuencia de su uso a pesar de lo necesarios, versátiles, útiles e intuitivos que resultan. Empezando por el final, el aprendizaje de la metodología de revisión sistemática y de los árboles de decisión no es más difícil que el de otros métodos de investigación y técnicas multivariadas que de manera tradicional se vienen incluyendo en los programas de formación de orientadores. Por otra parte, son recursos especialmente útiles en nuestra sociedad del conocimiento, porque ayudan a seleccionar información cuando se dispone de multitud de publicaciones o de datos, lo que resulta especialmente importante para tomar decisiones. Estos procedimientos son también idóneos para ser aplicados en un campo profesional en el que muchas veces no se cumplen los supuestos para trabajar con metodologías más fuertes. Por último, se presentan como necesarios porque solo puede crecer el corpus teórico de la orientación si, además de cumplir con los requisitos metodológicos que exijan los objetivos y las características de los datos, la investigación y la intervención que se realiza se sitúa en los hombros del gigante que representa lo que otros investigadores han aportado antes.

La propuesta, no obstante, cuenta con limitaciones. En este trabajo se integran, por su carácter eminentemente didáctico, dos estudios en una única investigación. Sin embargo, dado que la revisión sistemática es en sí misma una metodología de investigación, que puede enclavarse dentro de cualquiera de los paradigmas existentes, cualitativo, cuantitativo o mixto (Harden y Thomas, 2005), suele presentarse como un trabajo independiente. Aquí se ha podido incorporar una revisión sistemática como fase previa al estudio empírico porque se ha acotado excesivamente, limitando la búsqueda a ÍnDICEs-CSIC. Pero en una revisión sistemática con pretensiones de obtener un panorama más amplio habría que haber consultado bases como Dialnet, Scielo, Redalyc, Scopus, WOS o ERIC. Los trabajos de revisión son en realidad investigaciones secundarias que, si pretenden ser representativos de la evidencia hallada sobre un problema, en un contexto amplio y en un momento dado, exigen la consulta de múltiples bases y constituyen documentos científicos con entidad propia. Por otra parte, por razones de espacio y derivadas del propósito meramente ilustrativo de la revisión realizada aquí, se han obviado fases del proceso metodológico que en una revisión con fines más ambiciosos habrían de considerarse, como es la evaluación del peso de la evidencia de cada estudio o las garantías de calidad en que descansan los resultados de la síntesis (Andrews y Harlen, 2006). Estas fases son especialmente interesantes cuando el propósito de la revisión es la generación de evidencia acerca de la efectividad de una intervención. Otra limitación de la revisión realizada es que se han quedado fuera trabajos interesantes porque, también por razones de espacio, se filtraron y se excluyeron todos los estudios anteriores a 2016 y la búsqueda se limitó a unos descriptores muy concretos. Nuestro propósito, con esta propuesta, ha sido poner de manifiesto que, aunque se publiquen como investigaciones independientes, conviene que la revisión sea el punto de partida de una investigación primaria que pretenda ir un paso más allá en la frontera del conocimiento. El modo más viable y frecuente de hacerlo es redactar el estado de la cuestión utilizando revisiones publicadas sobre el tema, considerando este tipo de referencias como el modo más apropiado 
para aproximarse a un estado del arte fiable. En cuanto a la propuesta de realizar estudios empíricos con árboles de decisión, cuenta con la limitación de que se requieren bases de datos amplias, aunque los orientadores de centro o de equipo no son ajenos al manejo de grandes cantidades de datos.

Un inconveniente que puede derivarse de una lectura superficial de estas propuestas es que viene a sumar complejidad a la ya complicada tarea del orientador, quien se ve en la necesidad de desarrollar múltiples funciones disponiendo de medios muchas veces insuficientes. Pero una lectura con mirada larga nos lleva a la consideración de que, como en cualquier oficio, disponer de herramientas adecuadas es ventajoso, no solo para una intervención orientadora concreta e inmediata sino también y sobre todo para el desarrollo de la profesión. Los logros tan distintos a los que se llega en las diferentes ramas científicas están vinculados con las técnicas de investigación que en cada una se emplea y con el respeto que desde cada disciplina se tiene por la investigación anterior. En nuestro ámbito, a pesar del auge que va ganando el enfoque de Educación Basada en la Evidencia, todavía es importante la brecha que hay entre lo que resulta de la investigación y las creencias que marcan el proceder de los profesionales de la orientación. Este gap puede ser uno de los factores que expliquen el hecho de que la investigación que se realiza en orientación no produzca resultados más concluyentes que retroalimenten de manera más efectiva el quehacer profesional.

En consecuencia, como prospectiva, sería conveniente avanzar en el desarrollo del rol del orientador como investigador, 1) aportando síntesis cualitativas, cuantitativas o mixtas sobre otros temas de interés para la orientación educativa, que sirvan de anclaje para edificar la disciplina y sus procedimientos de intervención sobre cimientos sólidos (Camilli Trujillo et al., 2020) y 2) ensayando el uso de otras técnicas de minería de datos que faciliten al profesional el trabajo de interpretar la compleja realidad a la que se enfrenta a diario, ayudándole a separar el oro de la arena o la ganga de la mena (Asensio Muñoz et al., 2018; Lizasoain, 2012), esto es, llevándole a la obtención de conocimiento a partir del estudio de los datos.

El aprendizaje que supone la adopción en orientación del enfoque basado en la evidencia y la pericia metodológica que requiere es asumible desde un programa de formación de máster, marco en el cual este enfoque se erige como altamente interesante para la realización de los trabajos fin de máster (TFM). Y a su consolidación puede contribuir la realización de tesis doctorales con esta filosofía como denominador común.

\section{Referencias bibliográficas}

Alonso Ruido, P., Rodríguez Castro, Y., Lameiras Fernández, M. y Carrera Fernández, M.V. (2015). Hábitos de uso en las redes sociales de los y las adolescentes: Análisis de género. Revista de Estudios e Investigación en Psicología y Educación, 13, 54-57. https://doi.org/10.17979/reipe.2015.0.13.317

Álvarez García, D., García, T., Cueli, M. y Núñez, J.C. (2019). Control parental del uso de internet durante la adolescencia: Evolución y diferencias de género. Revista Iberoamericana de Diagnóstico y Evaluación, 2(51), 19-31. https://doi.org/10.21865/RIDEP51.2.02

Andrews, R. y Harlen, W. (2006). Issues in synthesizing research in Education. Educational Research, 48(3), 287-299. https://doi.org/10.1080/00131880600992330 
Arroyo Resino, D., Constante Amores, I.A. y Asensio Muñoz, I. (2019). Debate on grade repetition: An empirical study from PISA 2015. Educación XX1, 22(2), 69-92. https://doi.org/10.5944/educXX1.22479

Asensio Muñoz, I., Carpintero, E., López, E. y Expósito, E. (2018). How much gold is in the sand? Data mining with Spain's PISA 2015 results. Revista Española de Pedagogía, 270, 225-245. https://doi.org/10.22550/REP76-2-2018-02

Blanco, Á. (2012). Evidencia en educación. Normas, sistemas de calificación y prácticas de difusión adoptadas por algunas iniciativas institucionales. En M. Castro (Coord.), Elogio a la Pedagogía Científica. Un liber amicorum para Arturo de la Orden (pp. 55-72). Grafididma.

Blanco Blanco, Á., Asensio Muñoz, I., Carpintero Molina, E., Ruiz de Miguel, C. y Expósito Casas, E. (2017). Applications of hierarchical segmentation in measurement and evaluation of educational programs. Examples with a financial education program. Educación $X X 1,20(2)$, 235-257. https://doi.org/10.5944/educXX1.19039

Boza, A., Toscano, M. y Salas, M. (2007). ¿Qué es lo que hace un orientador? Roles y funciones del orientador en educación secundaria. XXI. Revista de Educación, 9, 111-131. http://www.uhu.es/publicaciones/ojs/index.php/xxi/article/view/520/756/520-121-1-PB.pdf

Cabero, J., Barroso, J., Llorente, M.C. y Yanes, C. (2016). Redes sociales y tecnologías de la información y la comunicación en educación: Aprendizaje colaborativo, diferencias de género, edad y preferencias. Revista de Educación a Distancia, 51, Art. 1. https://doi.org/10.6018/red/51/1

Calvo, S. y San Fabián, J.L. (2018). Redes sociales y socialización afectiva de las personas jóvenes: Necesidades docentes en educación secundaria obligatoria. Revista Iberoamericana sobre Calidad, Eficacia y Cambio en Educación, 16(2), 5-20. https://doi.org/10.15366/reice2018.16.2.001

Camilli, C. (2015). Aprendizaje cooperativo e individual en el rendimiento académico en estudiantes universitarios: Un meta-análisis (Tesis doctoral). Universidad Complutense de Madrid. https://eprints.ucm.es/30997/1/T36191.pdf

Camilli Trujillo, C., Arroyo Resino, D., Asensio Muñoz, I. y Mateos Gordo, P. (2020). Hacia la educación basada en la evidencia: un método y un tema. Revista Electrónica en Educación y Pedagogía, 4(6), 69-85. https://doi.org/10.15658/rev.electron.educ.pedagog20.05040606

Colás Bravo, P., Conde Jiménez, J. y Reyes de Cózar, S. (2017). Competencias digitales del alumnado no universitario. Revista Latinoamericana de Tecnología Educativa, 16(1), 7-20. https://medra.org/10.17398/1695-288X.16.1.7

De Frutos Torres, B. y Marcos Santos, M. (2017). Negative experiences and risk perception disconnection on the networking sites by teenagers. El profesional de la Información, 26(1), 88-96. https://doi.org/10.3145/epi.2017.ene.09

Donoso Vázquez, T., Rubio Hurtado, M.J. y Vilà Baños, R. (2018). Adolescence and gender violence 2.0: Concepts, behavior and experiences. Educación XX1, 21(1), 109-134. https://doi.org/10.5944/educXX1.20180

Fernández, J., Peñalva, A. y Irazabal, I. (2015). Hábitos de uso y conductas de riesgo en Internet en la preadolescencia. Comunicar, 22(44), 113-120. https://doi.org/10.3916/C44-2015-12

Giménez, A.M., Luengo, J.A. y Bartrina, M.J. (2017). ¿Qué hacen los menores en internet? Usos de las TIC, estrategias de supervisión parental y exposición a riesgos. Electronic Journal of Research in Educational Psychology, 15(3), 533-552. https://doi.org/10.14204/ejrep.43.16123 
Gomes, F. y Sendín, J.C. (2014). Internet como refugio y escudo social: Usos problemáticos de la Red por jóvenes españoles. Comunicar, 22(43), 45-53. https://doi.org/10.3916/C43-2014-04

González Sanmamed, M., Muñoz Carril, P.C. y Dans Álvarez de Sotomayor, I. (2017). Factors which motivate the use of social networks by students. Psicothema, 29(2), 204-210. https://doi.org/10.7334/psicothema2016.127

Grañeras, M. y Parras, A. (Coords.), (2009). Orientación Educativa: Fundamentos teóricos, modelos institucionales y nuevas perspectivas. MEC-CIDE.

Hernández Rivero, V.M. y Mederos Santana, Y. (2018). Papel del orientador/a educativo como asesor/a: Funciones y estrategias de apoyo. Revista Española de Orientación y Psicopedagogía, 29(1), 40-57. https://doi.org/10.5944/reop.vol.29.num.1.2018.23293

Harden, A. y Thomas, J. (2005). Methodological issues in combining diverse study types in systematic reviews. International Journal of Social Research Methodology, 8(3), 257-271. https://doi.org/10.1080/13645570500155078

Johnson, R.B. y Onwuegbuzie, A.J. (2004) Mixed methods research: A research paradigm whose time has come. Educational Researcher, 33(7), 14-26. https://doi.org/10.3102\%2F0013189X033007014

Kass, G.V. (1980). An exploratory technique for investigating large quantities of categorical data. Applied Statistics, 29(2), 119-127. https://doi.org/10.2307/2986296

Lizasoain, L. (2012). Las técnicas de minería de datos aplicadas a la investigación educativa. Arboles estadísticos de decisión. En M. Castro (Coord.), Elogio a la Pedagogía Científica. Un liber amicorum para Arturo de la Orden (pp. 101-121). Grafidridma.

Lizasoain, L. y Joaristi, L. (2000). El análisis de datos en la evaluación de programas educativos. Revista de Investigación Educativa, 18(2), 357-379.

López de Ayala, M.C., Martínez Pastor, E. y Catalina García, B. (2019). Nuevas estrategias de mediación parental en el uso de las redes sociales por adolescentes. El profesional de la Información, 28(5), e280523. https://doi.org/10.3145/epi.2019.sep.23

López Martín, E., Expósito Casas, E., Carpintero Molina, E. y Asensio Muñoz, I. (2018). What does PISA tell us about the teaching and learning of sciences? An approach through decision trees. Revista de Educación, 382, 133-161. https://doi.org/10.4438/1988-592X-RE-2018-382-395

Malo, S., Martín, M. y Viñas, F. (2018). Uso excesivo de redes sociales: Perfil psicosocial de adolescentes españoles. Comunicar, 26(56), 101-110. https://doi.org/10.3916/C56-2018-10

Martín Martín, M. (2015). Cambios psicosociales en los adolescentes. Incidencia del uso de las redes sociales (Tesis doctoral). Universidad Complutense de Madrid. https://eprints.ucm.es/37737/1/T37232.pdf

Martín-Martín, M., Asensio-Muñoz, I. y Bueno-Álvarez, J.A. (2021). Uso de las redes sociales en estudiantes de secundaria: análisis de perfiles para la intervención educativa. Revista Complutense de Educación, 32(3), 303-314. http://dx.doi.org/10.5209/rced.57189

Martín Montilla, A., Pazos Gómez, M., Montilla Coronado, M.V.C. y Romero Oliva, C. (2016). A current mode of gender violence in couples of young people: Social networks. Educación $X X 1$, 19(2), 405-429. https://doi.org/10.5944/educXX1.16473

Martínez Clares, P. (Coord.), (2017). Manual de orientación educativa y profesional. DM. 
Oberst, U., Chamarro, A. y Renau, V. (2016). Gender Stereotypes 2.0: Self-representations of Adolescents on Facebook. Comunicar, 24(48), 81-90. https://doi.org/10.3916/C48-2016-08

Orden, A., Garduño, L., Asensio, I., Mafokozi, J., González, C. y Muñoz, A. (2002). Funciones de la Universidad. Revista de Educación, 328, 267-281.

Parada Rico, D. y García Suárez, I. (2017). Adolescent fathers and mothers in the exercise of parenting. Revista Ciencia y Cuidado, 14(2), 97-113. https://doi.org/10.22463/17949831.1113

Pavón Arrizabalaga, A., Zubergoitia, A., Astigarraga, I. y Juaristi, P. (2016). Teenagers' Consumption of TV Series in the Era of TV Digitalization: Uses and Gratifications. Dígitos, 2, 35-51. https://revistadigitos.com/index.php/digitos/article/view/49/19/49-128-1-SM.pdf

Pérez Escoda, A. y Contreras, P. (2018). Smartphone y redes sociales para el desarrollo de competencias mediáticas y digitales en niños y adolescentes: Musical.ly. Aula Abierta, 47(3), 281-290. https://doi.org/10.17811/rifie.47.3.2018.281-290

Plaza, J. (2016). Impacto de las redes sociales virtuales en estudiantes adolescentes. Revista Internacional de Tecnologías en la Educación, 3(1), 53-63. https://doi.org/10.37467/gkarevedutech.v3.281

Prats, M.A., Torres, A., Oberst, U. y Carbonell, X. (2018). Design and application of educational workshops for the healthy use of the internet and online social networks in adolescents: Description of a pilot study. Píxel-Bit. Revista de Medios y Educación, 52, 111-124. https://doi.org/10.12795/pixelbit.2018.i52.08

Ramírez García, A., Salcines Talledo, I. y González Fernández, N. (2020). Los dispositivos móviles en el hogar. Interés formativo de las familias españolas. REOP, 31(1), 43-6. https://doi.org/10.5944/reop.vol.31.num.1.2020.27286

Ricoy, M.C. y Fernández Rodríguez, J. (2016). Leisure time and resources in adolescents. Educatio Siglo XXI, 34(2), 103-124. https://doi.org/10.6018/j/263831

Rodríguez García, L. y Magdalena Benedito, J.R. (2016). Perspective of Young People about Security and Privacy on Social Networks. Icono, 14, 24-49. https://doi.org/10.7195/ri14.v14i1.885

Rojas Jara, C., Henríquez, F., Sanhueza, F., Núñez, P., Inostroza, E., Solís, A. y Contreras, D. (2018). Internet addiction and use of social networks in adolescents: A review. Revista Española de Drogodependencias, 43(4), 39-54.

Romera, E.M., Cano, J.J., García-Fernández, C.M. y Ortega-Ruiz, R. (2016). Cyberbullying: Social Competence, Motivation and Peer Relationships. Comunicar, 24(48), 71-79. https://doi.org/10.3916/C48-2016-07

Rubiales, J., Russo, D., Paneiva, J.P. y González, R. (2018). Systematic review of socialemotional training programs for children and adolescents. Revista Costarricense de Psicología, 37(2), 163-186. https://doi.org/10.22544/rcps.v37i02.05

Ruido, P., Castro, Y. y Fernández, M. (2017). El impacto de las redes sociales en adolescentes gallegos/as: Análisis cualitativo. Revista de Estudios e Investigación en Psicología y Educación, Extr.(13), 52-56. https://doi.org/10.17979/reipe.2017.0.13.2281

Sabater, C. (2016). La influencia de la tecnología en la intimidad de los jóvenes: Hallazgos en la Comunidad Autónoma de La Rioja. Ar@cne, 214. http://www.ub.edu/geocrit/aracne/aracne214.pdf 
Sanz Oro, R. (2000). El orientador como investigador en la acción. Revista de Investigación Educativa, 18(2), 509-519

Shamseer, L., Moher, D., Clarke, M., Ghersi, D., Liberati, A., Petticrew, M., Shekelle, P. y Stewart, L.A. (2015). Preferred reporting items for systematic review and meta-analysis protocols (PRISMA-P) 2015: Elaboration and explanation. British Medical Journal, 350, g7647. https://doi.org/10.1136/bmi.g7647

Sobrado, L., Nogueira, M. y García, R. (2013). Las redes sociales de internet en el ámbito de la orientación educativa y profesional. En M.C. Cardona, E. Chiner y A. Giner (Eds.), Investigación e Innovación Educativa al Servicio de Instituciones y Comunidades Globales, Plurales y Diversas (pp. 494-503). Universidad de Alicante. https://www.uv.es/aidipe/congresos/Actas XVI Congreso.pdf

Vega Almeida, R.L. y Arencibia Jorge, R. (2019). The dark side of Internet: A bibliometric study of online addictive behaviors. Revista Española de Documentación Científica, 42(4), e251. https://doi.org/10.3989/redc.2019.4.S8

Villanueva Blasco, V.J. y Serrano Bernal, S. (2019). Patrón de uso de internet y control parental de redes sociales como predictor de sexting en adolescentes: Una perspectiva de género. Revista de Psicología y Educación, 14(1), 16-26. https://doi.org/10.23923/rpye2019.01.168

Villanueva-Baselga, S., Aguilar, C. y Sánchez, L. (2017). Participación Política, Medios de Comunicación y Redes Sociales en los Adolescentes Catalanes. International Journal of Sociology of Education, 6(1), 61-84. https://doi.org/10.17583/rise.2017.2496

Fecha de entrada: 31 enero 2020

Fecha de revisión: 6 noviembre 2020

Fecha de aceptación: 28 noviembre 2020 